\title{
Independent Sets in Graphs with an Excluded Clique Minor
}

\author{
David R. Wood" \\ Departament de Matemàtica Aplicada II \\ Universitat Politècnica de Catalunya \\ Barcelona, Spain \\ david.woodeupc.es
}

received December 7, 2006, accepted August 22, 2007.

Let $G$ be a graph with $n$ vertices, with independence number $\alpha$, and with no $K_{t+1}$-minor for some $t \geq 5$. It is proved that $(2 \alpha-1)(2 t-5) \geq 2 n-5$. This improves upon the previous best bound whenever $n \geq \frac{2}{5} t^{2}$.

Keywords: graph, minor, independent set, Hadwiger's Conjecture.

Mathematics Subject Classification: 05C15 (Coloring of graphs and hypergraphs)

\section{Introduction}

In 1943, Hadwiger [7] made the following conjecture, which is widely considered to be one of the most important open problems in graph theory (i)], see [19] for a survey.

Hadwiger's Conjecture. For every integer $t \geq 1$, every graph with no $K_{t+1}$-minor is $t$-colourable. That is, $\chi(G) \leq \eta(G)$ for every graph $G$.

Hadwiger's Conjecture is trivial for $t \leq 2$, and is straightforward for $t=3$; see [4, 7, 22]. In the cases $t=4$ and $t=5$, Wagner [20] and Robertson et al. [16] respectively proved that Hadwiger's Conjecture is equivalent to the Four-Colour Theorem [2, 3, 6, 15]. Hadwiger's Conjecture is open for all $t \geq 6$.

\footnotetext{
${ }^{\dagger}$ Research supported by a Marie Curie Fellowship from the European Commission under contract MEIF-CT-2006-023865, and by the projects MEC MTM2006-01267 and DURSI 2005SGR00692.

(i) All graphs considered in this note are undirected, simple and finite. Let $G$ be a graph with vertex set $V(G)$. Let $X \subseteq V(G)$. $X$ is connected if the subgraph of $G$ induced by $X$ is connected. $X$ is dominating if every vertex of $G \backslash X$ has a neighbour in $X . X$ is independent if no two vertices in $X$ are adjacent. The independence number $\alpha(G)$ is the maximum cardinality of an independent set of $G$. $X$ is a clique if every pair of vertices in $X$ are adjacent. The clique number $\omega(G)$ is the maximum cardinality of a clique in $G$. A $k$-colouring of $G$ is a function that assigns one of $k$ colours to each vertex of $G$ such that adjacent vertices receive distinct colours. The chromatic number $\chi(G)$ is the minimum integer $k$ such that $G$ is $k$-colourable. A minor of $G$ is a graph that can be obtained from a subgraph of $G$ by contracting edges. The Hadwiger number $\eta(G)$ is the maximum integer $n$ such that the complete graph $K_{n}$ is a minor of $G$.
}

1365-8050 @ 2007 Discrete Mathematics and Theoretical Computer Science (DMTCS), Nancy, France 
Progress on the $t=6$ case has been recently been obtained by Kawarabayashi and Toft [10] (without using the Four-Colour Theorem). The best known upper bound is $\chi(G) \leq c \cdot \eta(G) \sqrt{\log \eta(G)}$ for some constant $c$, independently due to Kostochka [11] and Thomason [17, 18].

Woodall [21] observed that since $\alpha(G) \cdot \chi(G) \geq|V(G)|$ for every graph $G$, Hadwiger's Conjecture implies that

$$
\alpha(G) \cdot \eta(G) \geq|V(G)| .
$$

Equation (1) holds for $\eta(G) \leq 5$ since Hadwiger's Conjecture holds for $t \leq 5$. For example, $\alpha(G) \geq$ $\frac{1}{4}|V(G)|$ for every planar graph $G$. It is interesting that the only known proof of this result depends on the Four-Colour Theorem. The best bound not using the Four-Colour Theorem is $\alpha(G) \geq \frac{2}{9}|V(G)|$ due to Albertson [1].

Equation (1) is open for $\eta(G) \geq 6$. In general, (1) is weaker than Hadwiger's Conjecture, but for graphs with $\alpha(G)=2$ (that is, graphs whose complements are triangle-free), Plummer et al. [13] proved that (1) is in fact equivalent to Hadwiger's Conjecture. The first significant progress towards (1) was made by Duchet and Meyniel [5] (also see [12]), who proved that

$$
(2 \alpha(G)-1) \cdot \eta(G) \geq|V(G)| .
$$

This result was improved by Kawarabayashi et al. [8] to

$$
(2 \alpha(G)-1) \cdot \eta(G) \geq|V(G)|+\omega(G) .
$$

Assuming $\alpha(G) \geq 3$, Kawarabayashi et al. [8] proved that

$$
(4 \alpha(G)-3) \cdot \eta(G) \geq 2|V(G)|,
$$

which was further improved by Kawarabayashi and Song [9] to

$$
(2 \alpha(G)-2) \cdot \eta(G) \geq|V(G)|
$$

The following theorem is the main contribution of this note.

Theorem 1 Every graph $G$ with $\eta(G) \geq 5$ satisfies

$$
(2 \alpha(G)-1)(2 \eta(G)-5) \geq 2|V(G)|-5 .
$$

Observe that Theorem 1 represents an improvement over (2), (4) and (5) whenever $\eta(G) \geq 5$ and $|V(G)| \geq \frac{2}{5} \eta(G)^{2}$. For example, Theorem 1 implies that $\alpha(G)>\frac{1}{7}|V(G)|$ for every graph $G$ with $\eta(G) \leq 6$, whereas each of (2), (4) and (5) imply that $\alpha(G)>\frac{1}{12}|V(G)|$.

\section{Proof of Theorem 1}

Theorem 1 employs the following lemma by Duchet and Meyniel [5]. The proof is included for completeness.

Lemma 1 ([5]) Every connected graph $G$ has a connected dominating set $D$ and an independent set $S \subseteq D$ such that $|D|=2|S|-1$. 
Proof: Let $D$ be a maximal connected set of vertices of $G$ such that $D$ contains an independent set $S$ of $G$ and $|D|=2|S|-1$. There is such a set since $D:=S:=\{v\}$ satisfies these conditions for each vertex $v$. We claim that $D$ is dominating. Otherwise, since $G$ is connected, there is a vertex $v$ at distance 2 from $D$, and there is a neighbour $w$ of $v$ at distance 1 from $D$. Let $D^{\prime}:=D \cup\{v, w\}$ and $S^{\prime}:=S \cup\{v\}$. Thus $D^{\prime}$ is connected and contains an independent set $S^{\prime}$ such that $\left|D^{\prime}\right|=2\left|S^{\prime}\right|-1$. Hence $D$ is not maximal. This contradiction proves that $D$ is dominating.

The next lemma is the key to the proof of Theorem 1 .

Lemma 2 Suppose that for some integer $t \geq 1$ and for some real number $p \geq t$, every graph $G$ with $\eta(G) \leq t$ satisfies $p \cdot \alpha(G) \geq|V(G)|$. Then every graph $G$ with $\eta(G) \geq t$ satisfies

$$
\alpha(G) \geq \frac{2|V(G)|-p}{4 \eta(G)+2 p-4 t}+\frac{1}{2} .
$$

Proof: We proceed by induction on $\eta(G)-t$. If $\eta(G)=t$ the result holds by assumption. Let $G$ be a graph with $\eta(G)>t$. We can assume that $G$ is connected. By Lemma $1, G$ has a connected dominating set $D$ and an independent set $S \subseteq D$ such that $|D|=2|S|-1$. Now $\alpha(G) \geq|S|=\frac{|D|+1}{2}$. Thus we are done if

$$
\frac{|D|+1}{2} \geq \frac{2|V(G)|-p}{4 \eta(G)+2 p-4 t}+\frac{1}{2} .
$$

Now assume that 6 does not hold. That is,

$$
|D| \leq \frac{2|V(G)|-p}{2 \eta(G)+p-2 t}
$$

Thus

$$
|V(G \backslash D)|=|V(G)|-|D| \geq \frac{(2 \eta(G)+p-2 t-2)|V(G)|+p}{2 \eta(G)+p-2 t} .
$$

Since $D$ is dominating and connected, $\eta(G \backslash D) \leq \eta(G)-1$. Thus by induction,

$$
\begin{aligned}
\alpha(G) \geq \alpha(G \backslash D) & \geq \frac{2|V(G \backslash D)|-p}{4 \eta(G \backslash D)+2 p-4 t}+\frac{1}{2} \\
& \geq \frac{2(2 \eta(G)+p-2 t-2)|V(G)|+2 p}{(2 \eta(G)+p-2 t)(4 \eta(G)-4+2 p-4 t)}-\frac{p}{4 \eta(G)-4+2 p-4 t}+\frac{1}{2} \\
& =\frac{2|V(G)|-p}{4 \eta(G)+2 p-4 t}+\frac{1}{2} .
\end{aligned}
$$

This completes the proof.

Lemma 3 Suppose that Hadwiger's Conjecture is true for some integer $t$. Then every graph $G$ with $\eta(G) \geq t$ satisfies

$$
(2 \eta(G)-t)(2 \alpha(G)-1) \geq 2|V(G)|-t
$$


Proof: If Hadwiger's Conjecture is true for $t$ then $t \cdot \alpha(G) \geq|V(G)|$ for every graph $G$ with $\eta(G) \leq t$. Thus Lemma2 2 with $p=t$ implies that every graph $G$ with $\eta(G) \geq t$ satisfies

$$
\alpha(G) \geq \frac{2|V(G)|-t}{4 \eta(G)-2 t}+\frac{1}{2}
$$

which implies the result.

Theorem 1 follows from Lemma 3 with $t=5$ since Hadwiger's Conjecture holds for $t=5$ [16].

\section{Concluding Remarks}

The proof of Theorem 1 is substantially simpler than the proofs of (3)-(5), ignoring its dependence on the proof of Hadwiger's Conjecture with $t=5$, which in turn is based on the Four-Colour Theorem. A bound that still improves upon (2), (4) and (5) but with a completely straightforward proof is obtained from Lemma 3 with $t=3$ : Every graph $G$ with $\eta(G) \geq 3$ satisfies $(2 \eta(G)-3)(2 \alpha(G)-1) \geq 2|V(G)|-3$.

We finish with an open problem. The method of Duchet and Meyniel [5] was generalised by Reed and Seymour [14] to prove that the fractional chromatic number $\chi_{f}(G) \leq 2 \eta(G)$. For sufficiently large $\eta(G)$, is $\chi_{f}(G) \leq 2 \eta(G)-c$ for some constant $c \geq 1$ ?

\section{References}

[1] Michael O. Albertson. A lower bound for the independence number of a planar graph. $J$. Combinatorial Theory Ser. B, 20(1):84-93, 1976.

[2] Kenneth Appel and Wolfgang HaKen. Every planar map is four colorable. I. Discharging. Illinois J. Math., 21(3):429-490, 1977.

[3] Kenneth Appel, Wolfgang Haken, and John Koch. Every planar map is four colorable. II. Reducibility. Illinois J. Math., 21(3):491-567, 1977.

[4] Gabriel A. Dirac. A property of 4-chromatic graphs and some remarks on critical graphs. $J$. London Math. Soc., 27:85-92, 1952.

[5] Pierre Duchet and Henri Meyniel. On Hadwiger's number and the stability number. Annals of Discrete Mathematics, 13:71-73, 1982.

[6] Georges Gonthier. A computer-checked proof of the four colour theorem. 2005. Microsoft Research, Cambridge, U.S.A.

[7] Hugo Hadwiger. Über eine Klassifikation der Streckenkomplexe. Vierteljschr. Naturforsch. Ges. Zürich, 88:133-142, 1943.

[8] Ken-IChi Kawarabayashi, Michael D. Plummer, and BJarne Toft. Improvements of the theorem of Duchet and Meyniel on Hadwiger's conjecture. J. Combin. Theory Ser. B, 95(1):152-167, 2005.

[9] Ken-IChi KaWarabayashi and ZI-XIa Song. Independence number and clique minors. $J$. Graph Theory, to appear. 
[10] Ken-ichi Kawarabayashi and Bjarne Toft. Any 7-chromatic graph has $K_{7}$ or $K_{4,4}$ as a minor. Combinatorica, 25(3):327-353, 2005.

[11] Alexandr V. KostochKa. The minimum Hadwiger number for graphs with a given mean degree of vertices. Metody Diskret. Analiz., 38:37-58, 1982.

[12] Frédéric Maffray and Henri Meyniel. On a relationship between Hadwiger and stability numbers. Discrete Math., 64(1):39-42, 1987.

[13] Michael D. Plummer, Michael Stiebitz, and Bjarne Toft. On a special case of Hadwiger's conjecture. Discuss. Math. Graph Theory, 23(2):333-363, 2003.

[14] Bruce Reed and Paul Seymour. Fractional colouring and Hadwiger's conjecture. J. Combin. Theory Ser. B, 74(2):147-152, 1998.

[15] Neil Robertson, Daniel P. Sanders, Paul D. Seymour, and Robin Thomas. The fourcolour theorem. J. Combin. Theory Ser. B, 70(1):2-44, 1997.

[16] Neil Robertson, Paul D. Seymour, and Robin Thomas. Hadwiger's conjecture for $K_{6}$-free graphs. Combinatorica, 13(3):279-361, 1993.

[17] Andrew Thomason. An extremal function for contractions of graphs. Math. Proc. Cambridge Philos. Soc., 95(2):261-265, 1984.

[18] Andrew Thomason. The extremal function for complete minors. J. Combin. Theory Ser. B, 81(2):318-338, 2001.

[19] BJarne Toft. A survey of Hadwiger's conjecture. Congr. Numer., 115:249-283, 1996.

[20] Klaus Wagner. Über eine Eigenschaft der ebene Komplexe. Math. Ann., 114:570-590, 1937.

[21] Douglas R. Woodall. Subcontraction-equivalence and Hadwiger's conjecture. J. Graph Theory, 11(2):197-204, 1987.

[22] Douglas R. Woodall. A short proof of a theorem of Dirac's about Hadwiger's conjecture. $J$. Graph Theory, 16(1):79-80, 1992. 
